\title{
Anorectal Melanoma
}

\author{
Amalia Stefanou, M.D. ${ }^{1}$ and Surya P.M. Nalamati, M.D. ${ }^{1}$
}

\begin{abstract}
Anorectal melanoma is a disease that can be difficult to diagnose because of its unclear presentation. After diagnosis, the main treatment available is surgical resection. Sentinel lymph node mapping has an unclear role in its management. Adjuvant therapy has long been recommended; however, there are no strong data to support its use. Prognostic factors to help gauge survival are also not clear; nevertheless, there is a strong association between stage of disease and histologic perineal invasion. Anorectal melanoma is a very rare disease with a dismal prognosis.
\end{abstract}

KEYWORDS: Anorectal melanoma, surgery, cancer

Objectives: After reading this article, the reader should be able to describe the clinical presentation of anorectal melanoma and understand the current treatment strategy.

The varied histology of the anal canal gives rise to an assortment of lesions, most curable by wide local excision or chemotherapy, but unfortunately anal melanoma has a very serious course and poor prognosis. Anorectal malignant melanoma accounts for 1 to $3 \%$ of all anal tumors and $0.3 \%$ of all melanoma. ${ }^{1}$ The incidence is about equal between men and women and presents most frequently in the $5^{\text {th }}$ to $6^{\text {th }}$ decade mostly in Caucasian patients. Anorectal melanoma is documented as the third most common location of melanoma, after cutaneous and ocular. The location of the lesion can be either at the anal canal or rectum, and most arise from the dentate line. Anorectal malignant melanoma spreads along submucosal planes; therefore, it is often beyond complete resection at the time of diagnosis. Etiology is unknown and surgery is the mainstay of treatment. Chemotherapy and radiation therapy alone have not been shown to be effective, but may provide some benefit when used in an adjuvant fashion. Other new novel treatments may improve clinical outcome.

\section{ETIOLOGY}

All melanomas originate from the melanocyte, which is derived from embryologic neural crest cells. These cells are located throughout in the body, including eyes and mucosa surfaces, but are mainly in the epidermis. In the rectum, melanocytes are located at the anal transition zone and squamous zone. Melanocytes may undergo malignant transformation when exposed to ultraviolet $\mathrm{B}$ light, which is a carcinogenic stimulus. This relationship has been clearly seen in peripheral cutaneous melanoma, but is more difficult to understand the pathogenesis in anorectal melanoma. There is some data to suggest a role in immunology in the development of anorectal melanoma. There seems to be higher incidence in patients with HPV and also HIV.

Cutaneous melanoma makes up over $90 \%$ of cases of malignant melanoma. The next most common malignant melanoma is ocular. Anorectal melanoma comprises $<2 \%$ of all diagnosed melanomas. ${ }^{2}$

Anorectal malignant melanoma tends to drain submucosally to the inguinal and inferior mesenteric

Reickert, M.D.

Clin Colon Rectal Surg 2011;24:171-176. Copyright (C) 2011 by Thieme Medical Publishers, Inc., 333 Seventh Avenue, New York, NY 10001, USA. Tel: +1(212) 584-4662.

DOI: http://dx.doi.org/10.1055/s-0031-1286001.

ISSN 1531-0043.
${ }^{1}$ Division of Colon and Rectal Surgery, Department of Surgery, Henry Ford Hospital, Detroit, Michigan.

Address for correspondence and reprint requests: Surya P.M. Nalamati, M.D., Division of Colon and Rectal Surgery, Department of Surgery, Henry Ford Hospital, 2799 W. Grand Blvd., Detroit, MI 48202 (e-mail: snalama1@hfhs.org).

Uncommon Colorectal Neoplasms; Guest Editor, Craig A. 
lymph nodes, then the hypogastric, paraaortic lymph nodes. Because of this indolent pattern, at the time of diagnosis most patients have metastatic disease present and are beyond surgical cure. Additional locations of metastasis are the liver, lung, and brain., ${ }^{2,3}$ Of all patients diagnosed with anorectal melanoma the 5-year survival rate can range from 16 to $34 \%{ }^{4-7}$ In patients who have metastasis at the time of diagnosis, the disease-free survival rate may drop to $16 \%$ from $22 \%{ }^{6}$

\section{CLINICAL PRESENTATION AND DIAGNOSIS}

Anorectal malignant melanoma can be a very difficult diagnosis to make because of nonspecific symptoms, as well as its anatomic location. Patients tend to present late and clinicians may have a lower suspicion due to its rare incidence. The most common presenting complaints are bleeding in $55 \%$ of patients. Additionally, $34 \%$ of patients will complain of a perianal or rectal mass, and $13 \%$ will complain of pain. ${ }^{6}$ Other symptoms include pruritis, tenesmus, prolapsed hemorrhoid, change in stool habits and diarrhea. ${ }^{2,3,8}$ Patients who have metastasis at the time of presentation may additionally have fatigue, weight loss, and anemia. ${ }^{3}$

As with any workup for lower gastrointestinal (GI) bleeding, a colonoscopy should be done. Anorectal melanoma is frequently misdiagnosed as a thrombosed hemorrhoid, ${ }^{9}$ and so it is imperative to have a high index of suspicion and to fully evaluate all complaints of lower GI bleeding and pain. A colonoscopy will allow for evaluation of the tumor-look for synchronous lesions and obtain a tissue biopsy. A tissue biopsy is important especially to diagnose amelanotic melanoma. ${ }^{8}$ Once a mass is identified, endoscopic endorectal ultrasound may be considered to evaluate tumor thickness and surrounding nodal status. ${ }^{10}$ A computed tomography (CT) scan of the abdomen and pelvis is frequently utilized to determine if there is lymphadenopathy or metastasis to the liver, lung, or pelvis. At the time of diagnosis, 26 to $38 \%$ of patients will have metastatic disease present. ${ }^{6,11,12}$

Histologic markers can also be used in the diagnosis of anorectal melanoma. The presence of melanin can be helpful, but is clearly not reliably present in anorectal disease compared with cutaneous. Only 30 to $70 \%$ of anorectal malignant melanomas are amelanotic, making clinical and histopathologic diagnosis more difficult. ${ }^{4,13,14}$ The histologic markers of S-100, HMB-45, and vimentin are frequently identified and help clarify the diagnosis. ${ }^{5,8,10}$ Another study found a trend of longer survival in patients with an S-phase fraction of $<10 \%$ and lower $\mathrm{K}_{\mathrm{i}}-67$ and PCNA (proliferating cell nuclear antigen) scores. ${ }^{15,16}$ Histologic factors associated with a poor prognosis are perineural invasion and depth of invasion.
Table 1 American Joint Commission on Cancer Staging System for Anorectal Melanoma ${ }^{17}$

\begin{tabular}{lll}
\hline Stage & Spread & Depth $(\mathbf{m m})$ \\
\hline IA & Localized & 0.75 \\
IB & Localized & $0.76-1.5$ \\
IIA & Localized & $1.5-4.0$ \\
IIB & Localized & $>4.0$ \\
III & Regional nodes & $x$ \\
IV & Distant metastasis & $x$ \\
\hline
\end{tabular}

There are two methods of staging in anorectal melanoma. The American Joint Commission on Cancer has developed a staging method based on presence of tumor in lymph nodes and depth of primary tumor (Table 1$).{ }^{17}$

Another staging system is based only on disease spread. It describes local disease only as stage 1 , regional lymph node disease as stage 2 , and metastatic disease as stage $3 .^{3}$

\section{TREATMENT}

A multimodal coordination of treatment provides the best treatment and long-term outcomes in anorectal melanoma. Surgical resection is always recommended; adjuvant chemotherapy, immune therapy, and radiation have a limited role in curing the disease. There is strong data supporting the treatment of cutaneous melanoma based on thickness of tumor (Clark-Breslow ${ }^{18,19}$ ), but these guidelines have not been found to be applicable to anorectal melanoma disease.

\section{Surgery}

Despite the various modalities available for the treatment of anorectal melanoma, surgery has been a mainstay and the most effective means of control. The controversy has been whether abdominoperineal resection (APR) is needed or wide local excision (WLE) is adequate for complete treatment. Unfortunately, because anorectal melanoma is so rare, there have been no randomized controlled trials to evaluate the difference. Additionally, very few patients present with stage 1 disease to allow trials to occur. Patients who present with regional spread are found to have a 13-month survival; those with localized disease still only survive 34 months despite the therapy provided. ${ }^{11}$ Despite having limited disease, 8 of 13 patients that underwent complete resection died from disease progression at a mean of 24 months. ${ }^{12}$ Anorectal melanoma tends to recur early with distant metastasis and aggressively, so it important to take the morbidity and the patient's quality of life into consideration

Abdominal perineal resection, although a highly morbid operation, has long been thought to be the best 
means of control of anorectal melanoma. In 1997 there was a paradigm shift after two major studies found minimal improvement in survival. Retrospective studies were done by Brady et $\mathrm{al}^{13}$ and Thibault et al, ${ }^{6}$ which looked at a combined 135 patients with anal melanoma. One hundred eight patients underwent curative attempt by either APR or WLE and their outcomes recorded. Patients in both studies had uniformly poor survival rates. In a study from Brady et $\mathrm{al}^{13}$ at Memorial SloanKettering in New York, the APR group did have a longer survival rate, but this was not statistically significant. In a study from Thibault et al, ${ }^{6} 37$ patients had curative resection but still no significant survival difference was found between WLE to APR when comparing disease stage and 5-year survival. ${ }^{8}$ Before $1997,70 \%$ of all patients underwent APR and after 1997, 80\% would undergo WLE. ${ }^{7}$ Median disease-specific survival (DSS) was 39 months and the 5 -year DSS rate was only $34 \%$. Of patients with curative resection of any type, $74 \%$ recurred at one year with distant metastasis with or without locoregional recurrence despite operation. It was realized that WLE may be adequate to control disease while minimizing the morbidity of surgery because most patients do not have a significant long-term survival. A palliative APR or WLE with extensive disease may improve quality of life, but will not affect survival.

For curative intent, patients with stage 0 and 1 should have a WLE, but higher stage patients should have an APR. ${ }^{5}$ Others consider APR for smaller tumors with the goal of complete cure; however, the statistics are still not significant in terms of local recurrence and survival. ${ }^{13}$ Completion of lymphadenectomy during APR may not affect survival. ${ }^{7}$ However for stage IV patients, an APR may improve quality of life from chronic bleeding or obstruction from tumor. ${ }^{5}$ Abdominal perineal resection seems to better control local disease, but does not change the incidence of distant metastasis or survival. ${ }^{20}$ This suggests that anorectal melanoma is a systemic disease at the time of diagnosis and no surgical treatment, regardless how aggressive, will truly change the outcome. ${ }^{6}$

As indicated, a WLE can provide tumor control and sometimes cure in patients. Wide local excision is defined as a sphincter-saving operation with a defined margin around the tumor in two dimensions. The depth should be restricted to the subcutaneous compartment. The excision should be performed up to the internal sphincter muscle and side margin of $2 \mathrm{~cm}$ to the tumor. ${ }^{12}$ General resection margins recommended are: $1 \mathrm{~mm}$ tumor $=1 \mathrm{~cm}$ margin; 1 to $4 \mathrm{~mm}$ tumor $=2 \mathrm{~cm}$ margin . If the tumor is $>4 \mathrm{~mm}$, an APR is recommended. ${ }^{10} \mathrm{~A}$ $2 \mathrm{~cm}$ margin regardless of primary tumor size is recommended. ${ }^{14}$ The benefits of a WLE are quicker recovery, no need for a stoma, and minimal impact on bowel function. ${ }^{10}$ There is no value in a prophylactic lymph node dissection during a WLE, even when there are clinically positive nodes. ${ }^{10}$ Of patients who had a WLE, $62.5 \%$ had a recurrence, which is a rate comparable to APR. ${ }^{12}$ One center found that there was no difference in recurrence from WLE and APR; they also completed reexcision for recurrence after APR. ${ }^{21}$ WLE may not always be possible, for example, when tumor is invading the sphincter complex or when tumors are causing chronic bleeding or obstruction. ${ }^{8}$ Tumor depth is the strongest predictor of whether patients will undergo APR or WLE, so postoperative complications are found accordingly. ${ }^{12}$ This also conveys a selection bias. Either way, patients must be informed of risk of local recurrence and failure, as well as the potential need for further surgery in the future.

Patients that undergo a curative operation, either an APR or WLE, will have a better outcome than palliative operation alone $(P<.001)$ showing that despite metastatic disease, if an adequate operation is done, long-term survival is possible. ${ }^{5}$ In one of the largest retrospective reviews, a survival of 21 months was found for patients who underwent WLE and 17 months for patients who had an APR. ${ }^{22}$ The WLE had a higher rate of recurrence, but overall there was no difference in survival. The patients who had an APR did have a lower postoperative quality of life; the authors recommended a WLE whenever possible because it does not impact survival. It is difficult to compare patients who have had an APR or WLE because there are not any data that can compare prognostic indicators preoperatively. There is a selection bias in the series because surgeons will do a WLE more frequently in patients with a lower tumor thickness hoping for cure, while others will still attempt curative APR.

The goal of surgical management should be to maximize both quality and quantity of life because cure is so difficult with such an aggressive disease. Because there is no consensus survival advantage to performing an APR, the majority of the literature recommends WLE whenever possible to minimize morbidity. Abdominal perineal resection, although a more invasive operation, does not convey a survival benefit; hence, a WLE with sphincter preservation should be advised whenever possible. ${ }^{6,8}$ A WLE with negative margins, or an APR when WLE is not possible, should be recommended for patients with anorectal melanoma.

\section{Sentinel Lymph Node Mapping and Lymph Node Dissection}

The role of sentinel lymph node mapping has revolutionized the treatment and prognosis of breast cancer as well as cutaneous melanoma management. In these two diseases, it has been proven to affect operative treatment as well as predict outcomes. It has been recommended in the management of anorectal melanoma to evaluate for 
metastatic disease, but there are still little data to support what to do with the obtained information. Finding positive lymph node metastasis is important to identify metastatic disease and prevent understaging of primary tumor. The literature is still unclear on whether nodal metastasis will affect or predict long-term outcome, so its operative planning should be based on positive nodal metastasis. Because anorectal melanoma may metastasize to inguinal lymph nodes or mesenteric lymph nodes, sentinel lymph node biopsy is thus feasible. ${ }^{23,24}$ Sentinel lymph nodes were successfully found in four patients in two studies, three in the inguinal region and two in the presacral space. Lymphoscintigraphy was used preoperatively and blue dye intraoperatively. Only one patient had a positive sentinel lymph node for metastatic disease, but this demonstrated the capability of evaluating a sentinel lymph node for metastasis, particularly if all potential metastasis travels to one side where the lymph node of note is located. This would enable clear operative planning if a positive node was shown to affect outcome based on doing a lymph node dissection on a particular side. ${ }^{10}$ More research needs to be done to evaluate whether operative planning should be based on a sentinel lymph node. A complete lymph node dissection is generally recommended when positive nodal disease is present. However, because the prognosis with and without complete dissection is not significantly longer and it can increase the morbidity of operation, more and careful evaluation is needed to assess its effect on survival and quality of life.

\section{Adjuvant Therapy}

There is no current adjuvant therapy dictated as the standard of care in anorectal melanoma, particularly in metastatic disease. The medications used in malignant cutaneous melanoma may have some utility in anorectal melanoma. Some studies suggest that patients with regional node metastasis can expect a reasonable longterm survival after curative APR and adjuvant chemotherapy. ${ }^{5,20}$ However, there have not been any randomized controlled trials to specifically study this; therefore, they are of uncertain utility. It is clear, however, that chemotherapy alone without surgery provides no benefit in the treatment. ${ }^{8}$ There are data that suggest a positive correlation between patients who have adjuvant therapy and a prolonged DSS when compared with patients who only underwent surgery alone. ${ }^{5}$ The medications used in adjuvant therapy are cisplatin, vinblastine, dacarbazine, interferon B, and IL-2. ${ }^{8}$ Dacarbazine is the most commonly used single agent and usually initiates a partial response in $20 \%$ of patients in 4 to 6 months after treatment ${ }^{25}$; however there are no randomized prospective trials that compare it to placebo. Another combination of chemotherapy is known as the Dartmouth regimen: dacarbazine cisplatin carmustine, and tamox- ifen. ${ }^{26}$ It was studied in 240 patients and compared with dacarbazine alone. This combination demonstrated an improvement in response rate to therapy, but was not statistically significant and patients in both treatment arms still only had a median survival of 7 months. As expected, typical toxicities of chemotherapy are bone marrow suppression, nausea, vomiting, and fatigue. Radiation has not been shown to provide any benefit, except occasionally in palliative care for unresectable tumors.

Primary melanoma has a higher incidence in patients who are immunocompromised and though these patients have a higher incidence of all malignancies, this link has been the basis of investigation of the tumors strong susceptibility to immune assault. On histopathologic exam, anorectal melanoma typically is infiltrated with lymphocytes. Some patients with metastatic melanoma have been shown to respond to therapy with interferon $\alpha$ and interleukin-2, suggesting that metastatic melanoma is susceptible to immune assault. As a result, there have been investigations into target treatment strategies focusing on the immunologic components of the disease, including vaccines.

\section{PROGNOSIS}

Survival of patients with anorectal melanoma is uniformly poor. Despite many attempts to understand risk factors affecting prognosis, the incidence is so rare that a consensus of risk factors and outcome predictors has not been possible. ${ }^{12}$ Stage of melanoma at diagnosis and tumor thickness was the most predictive of outcome and not necessarily operation-performed. ${ }^{27,28}$ The mean survival of patients with anorectal melanoma after treatment is 20 months $^{8}$; there have been no long-term survivors among patients with stage 2 and stage 3 disease. ${ }^{3}$ The only preoperative factor found to have an association with improved survival is female gender. Most patients who have advanced disease and prolonged survival are women, but this might present a selection bias. $^{3}$ Women are more likely to have medical care and obtain close medical follow-up; however, there are no randomized controlled trials to examine this. Incidental discovery of tumor on symptomatic mass workup found a positive correlation with a lower disease specific survival $(P<.062){ }^{7}$ Five patients that were found to have anorectal melanoma incidentally while being treated for hemorrhoids all died of metastasis within 29 months and had a worse outcome compared with similar patients. ${ }^{6,7}$

As expected, patients who present without lymph node metastasis will have a survival advantage. Patients without lymph node metastasis have a 5-year survival rate of $20 \%$ versus $0 \%$ in patients with metastasis. ${ }^{13}$ Because of this finding, an APR was recommended for any patients with a tumor $<2 \mathrm{~mm}$ deep without lymph 
node metastasis for a better chance of cure and hopefully a longer survival. For patients with more advanced disease, WLE is recommended to minimize morbidity because there is no effect on survival. Another study did not find an association with survival in regional lymph node metastasis. ${ }^{7}$ This is counterintuitive because it is a strong predictor in cutaneous melanoma.

Primary tumor size $>2 \mathrm{~cm}$ is associated with a shorter DSS $(P<.23)$ and has a hazard ratio of 1.1. Tumor thickness is another component that has a strong relationship with disease-specific survival. Patients with a primary tumor $<2 \mathrm{~mm}$ were alive at 12 -years posttreatment, whereas patients with tumor $>2 \mathrm{~mm}$ all experienced recurrence. ${ }^{27}$ Tumor thickness $>10 \mathrm{~mm}$ had an association with a shorter disease-specific survival $(P<.08){ }^{7}$

There are many histologic markers that have had association with prognosis. A worse prognosis was associated with tumor thickness, tumor necrosis, and perineural invasion. ${ }^{7,10,13}$ Interestingly, tumor ulceration and melanin in situ melanoma had no effect on outcome. ${ }^{7}$ Peripheral nerve involvement had the strongest association with poor disease-specific survival, with all patients having recurrent disease within one year $(P<.01){ }^{7,10}$ The median survival with peripheral nerve involvement was 19 months compared with 39 months in patients without nerve involvement (hazard ratio =3.3). At one year, all but one patient had died and those remaining had widespread metastasis. The presence of tumor necrosis was also a poor histologic feature associated with disease recurrence. In one study, all six patents with tumor necrosis had tumor recurrence compared with $73 \%$ in patients without tumor necrosis $(P<.96){ }^{7}$ The histologic type of tumor had an association with recurrence, but not disease survival. Pure epithelioid histology was more likely to recur when compared with other histologies. ${ }^{7}$

\section{CONCLUSION}

Anorectal melanoma is a rare malignancy that differs a great deal from its counterpart of cutaneous melanoma. It is a difficult disease to not only diagnose but also treat. It is aggressive in its course and surgery is the main option for treatment. The prognosis is very poor, and in many cases remains poor despite mode of operation or therapy attempted.

\section{REFERENCES}

1. Mason JK, Helwig EB. Ano-rectal melanoma. Cancer 1966; 19(1):39-50

2. Moore HG, Guillem JG. Anal neoplasms. Surg Clin North Am 2002;82(6):1233-1251

3. Singer M, Mutch MG. Anal melanoma. Clin Colon Rectal Surg 2006;19(2):78-87
4. Chiu YS, Unni KK, Beart RW Jr. Malignant melanoma of the anorectum. Dis Colon Rectum 1980;23(2):122-124

5. Ishizone S, Koide N, Karasawa F, et al. Surgical treatment for anorectal malignant melanoma: report of five cases and review of 79 Japanese cases. Int J Colorectal Dis 2008;23(12): $1257-1262$

6. Thibault C, Sagar P, Nivatvongs S, Ilstrup DM, Wolff BG. Anorectal melanoma-an incurable disease?. Dis Colon Rectum 1997;40(6):661-668

7. Yeh JJ, Shia J, Hwu WJ, et al. The role of abdominoperineal resection as surgical therapy for anorectal melanoma. Ann Surg 2006;244(6):1012-1017

8. Yap LB, Neary P. A comparison of wide local excision with abdominoperineal resection in anorectal melanoma. Melanoma Res 2004;14(2):147-150

9. Felz MW, Winburn GB, Kallab AM, Lee JR. Anal melanoma: an aggressive malignancy masquerading as hemorrhoids. South Med J 2001;94(9):880-885

10. Stoidis CN, Spyropoulos BG, Misiakos EP, Fountzilas CK, Paraskeva PP, Fotiadis CI. Diffuse anorectal melanoma; review of the current diagnostic and treatment aspects based on a case report. World J Surg Oncol 2009;7:64

11. Podnos YD, Tsai NC, Smith D, Ellenhorn JD. Factors affecting survival in patients with anal melanoma. Am Surg 2006;72(10):917-920

12. Weyandt GH, Eggert AO, Houf M, Raulf F, Bröcker EB, Becker JC. Anorectal melanoma: surgical management guidelines according to tumour thickness. Br J Cancer 2003;89(11):2019-2022

13. Brady MS, Kavolius JP, Quan SH. Anorectal melanoma. A 64-year experience at Memorial Sloan-Kettering Cancer Center. Dis Colon Rectum 1995;38(2):146-151

14. Ward MW, Romano G, Nicholls RJ. The surgical treatment of anorectal malignant melanoma. Br J Surg 1986;73(1):68-69

15. Ben-Izhak O, Bar-Chana M, Sussman L, et al. Ki67 antigen and PCNA proliferation markers predict survival in anorectal malignant melanoma. Histopathology 2002;41(6):519-525

16. Ben-Izhak O, Levy R, Weill S, et al. Anorectal malignant melanoma. A clinicopathologic study, including immunohistochemistry and DNA flow cytometry. Cancer 1997;79(1): $18-25$

17. Chang AE, Karnell LH, Menck HR; The American College of Surgeons Commission on Cancer and the American Cancer Society. The National Cancer Data Base report on cutaneous and noncutaneous melanoma: a summary of 84,836 cases from the past decade. Cancer 1998;83(8): 1664-1678

18. NIH Consensus Conference. Diagnosis and treatment of early melanoma. JAMA 1992;268(10):1314

19. Cohn-Cedermark G, Rutqvist LE, Andersson R. Long-term results of a randomized study by the Swedish Melanoma Study Group on $2-\mathrm{cm}$ versus $5-\mathrm{cm}$ resection margins for patients with cutaneous melanoma with a tumor thickness of 0.8-2.0 mm. Cancer 2000;89(7):1495

20. Ballo MT, Gershenwald JE, Zagars GK, et al. Sphinctersparing local excision and adjuvant radiation for anal-rectal melanoma. J Clin Oncol 2002;20(23):4555-4558

21. Bullard KM, Tuttle TM, Rothenberger DA, et al. Surgical therapy for anorectal melanoma. J Am Coll Surg 2003; 196(2):206-211

22. Droesch JT, Flum DR, Mann GN. Wide local excision or abdominoperineal resection as the initial treatment for anorectal melanoma? Am J Surg 2005;189(4):446-449 
23. Olsha O, Mintz A, Gimon Z, et al. Anal melanoma in the era of sentinel lymph node mapping: a diagnostic and therapeutic challenge. Tech Coloproctol 2005;9(1):60-62

24. Sanli Y, Turkmen C, Kurul S, Ta F, Mudun A, Cantez S. Sentinel lymph node biopsy for the staging of anal melanoma: report of two cases. Ann Nucl Med 2006;20(9):629-631

25. Crosby T, Fish R, Coles B, Mason MD. Systemic treatments for metastatic cutaneous melanoma. Cochrane Database Syst Rev 2000;(2):CD001215

26. Chapman PB, Einhorn LH, Meyers ML, et al. Phase III multicenter randomized trial of the Dartmouth regimen versus dacarbazine in patients with metastatic melanoma. J Clin Oncol 1999;17(9):2745-2751

27. Wanebo HJ, Fortner JG, Woodruff J, MacLean B, Binkowski E. Selection of the optimum surgical treatment of stage I melanoma by depth of microinvasion: use of the combined microstage technique (Clark-Breslow). Ann Surg 1975;182(3):302-315

28. Slingluff CL Jr, Vollmer RT, Seigler HF. Anorectal melanoma: clinical characteristics and results of surgical management in twenty-four patients. Surgery 1990;107(1): 1-9 